\title{
Role of XRCC1 and ERCC5 polymorphisms on clinical outcomes in advanced non-small cell lung cancer
}

D. Liu, J. Wu, G.Y. Shi, H.F. Zhou and Y. Yu

Department of Medical Oncology, The Third Affiliated Hospital of Harbin Medical University, Harbin, China

Corresponding author: $\mathrm{Y} . \mathrm{Yu}$

E-mail: yanyu_hmu@163.com

Genet. Mol. Res. 13 (2): 3100-3107 (2014)

Received February 6, 2013

Accepted July 31, 2013

Published April 17, 2014

DOI http://dx.doi.org/10.4238/2014.April.17.6

ABSTRACT. We aimed to assess the role of polymorphisms of the
XRCC1 Arg194Trp, XRCC1 Arg399Gln, ERCC5 His1104Asp, and
ERCC5 His46His genes on clinical outcomes of advanced non-small cell
lung cancer (NSCLC) patients receiving platinum-based chemotherapy
regimens. A total of 378 NSCLC patients were asked to participate
within 1 month after diagnosis between January 2005 and January 2006 ,
and they were followed up until November 2011 . Genomic DNA of the
four genes was extracted using the Qiagen Blood Kit. Results showed
that individuals with XRCC1 399A/A and ERCC5 46T/T genotypes
were more likely to show positive responses to chemotherapy, with odds
ratio (OR) $=2.27$ and $95 \%$ confidence interval (CI) $=1.64-6.97$, and OR
$=1.90$, CI $=1.10-3.28$, respectively. The XRCC1 399 A/A genotype was
significantly associated with longer progression-free survival (PFS) and
overall survival (OS) rates, and the hazard ratios (HRs) (95\%CI) were
$0.48(0.25-0.88)$ and 0.51 ( $0.26-0.98)$, respectively. Similarly, NSCLC
patients carrying the ERCC5 $46 \mathrm{~T} / \mathrm{T}$ genotype were more likely to show
increased PFS and OS, with HRs $(95 \%$ CI) of 0.47 ( $0.22-0.82)$ and 0.52
$(0.31-0.96)$, respectively. In conclusion, our study indicated that XRCC1 
Arg399Gln and ERCC5 His46His might significantly influence the response to chemotherapy, and the two genetic polymorphisms are suggested to be routinely detected to determine NSCLC patients that are more likely to benefit from chemotherapy.

Key words: SNPs; XRCC1; ERCC5; Non-small cell lung cancer

\section{INTRODUCTION}

Lung cancer is the leading cause of cancer-related deaths throughout the world (IARC, 2008). Non-small cell lung cancer (NSCLC) is the primary lung cancer type, and is expected to account for $80 \%$ of all lung cancer cases. More than $70 \%$ of NSCLC patients present locally advanced or metastatic disease at the time of diagnosis due to the asymptomatic nature of early disease and the lack of effective screening modalities (William Jr. et al., 2009). It is estimated that the five-year survival rate for NSCLC is less than $15 \%$, and NSCLC has been reported as fatal cancer in China in the last 10 years (IARC, 2008). Since curative surgery is not an effective treatment for NSCLC, chemotherapy has become the main treatment measure for advanced NSCLC patients. Platinum-based doublets chemotherapy is presently the standard first-line chemotherapy used for advanced NSCLC patients. However, the response rate to platinum-based regimens in advanced NSCLC cases is approximately $20 \%$, and the median survival is approximately 7 to 9 months (Grønberg et al., 2009; Tahara et al., 2010). If patients show non-response to chemotherapy, tumor growth is not controlled, and the life of patients cannot be prolonged. Therefore, it would be more efficient to select patients who would best respond to chemotherapy to receive treatment, and thus improve the efficacy of chemotherapy in advanced NSCLC.

Efficiency of DNA damage repair systems is considered to be one of the most important mechanisms affecting inter-individual differences in the response to chemotherapy and the clinical outcome of patients. Damage induced by platinum agents is predominantly repaired by nucleotide excision repair (NER), base excision repair, and double-strand break repair pathways. NER is the main pathway for repairing platinum-DNA adducts, and involves the coordinated activity of more than 20 enzymes that act to remove and restore a segment of DNA containing bulky adducts (Reed, 1998; de Boer and Hoeijmakers, 2000). The X-ray repair cross-complementing protein 1 (XRCC1) is an indispensable component in base excision repair and single-strand break repair systems, and thus might play an important role in resistance to a variety of DNA-damaging agents. Two main single nucleotide polymorphisms (SNPs) in the XRCC1 gene, including nucleotide substitutions of C to T (Arg194Trp) and A to G (Arg399Gln), may be associated with the suboptimal DNA repair capacity of patients with NSCLC (Lunn et al., 1999; Weaver et al., 2005). The excision repair cross-complementing protein 5 (ERCC5) is another important component of NER, and nucleotide substitutions of $\mathrm{G}$ to $\mathrm{A}$ (His1104Asp) and $\mathrm{C}$ to $\mathrm{T}$ (His46His) have been associated with a significantly reduced risk of NSCLC (Cheng et al., 2000; Jeon et al., 2003).

In the present study, we prospectively evaluated whether polymorphisms in XRCC1 Arg194Trp, XRCC1 Arg399Gln, ERCC5 His1104Asp, and ERCC5 His46His genes could predict the prognosis of advanced NSCLC patients who received platinum-based chemotherapy. 


\section{MATERIAL AND METHODS}

\section{Subjects}

From January 2005 to January 2006, 378 patients who were diagnosed with primary NSCLC within 1 month were asked to participate in our study. All patients were selected from the Third Affiliated Hospital of Harbin Medical University, and diagnoses were confirmed with histological or cytological analysis. Patients who had a prior history of malignancy, an already cured tumor, or a history of chemotherapy, radiotherapy, or surgery were excluded from the study.

Socio-demographic characteristics and clinical characteristics were collected from medical records from doctors and nurses, including smoking, family history of cancer, clinical stage, and histology. All patients received platinum-based chemotherapy as the first-line treatment. The chemotherapeutic regimens included $75 \mathrm{mg} / \mathrm{m}^{2}$ cisplatin on the first day plus $1250 \mathrm{mg} / \mathrm{m}^{2}$ gemcitabine on the first and eighth days, $75 \mathrm{mg} / \mathrm{m}^{2}$ docetaxel on the first day, $25 \mathrm{mg} / \mathrm{m}^{2}$ vinorelbine on the first and eighth days, or $150 \mathrm{mg} / \mathrm{m}^{2}$ paclitaxel on the first day. The treatment was repeated every 3 weeks for a maximum of six cycles. If patients presented progressive disease or experienced unacceptable toxicity levels, the treatment was stopped. If patients showed Grade 3 non-hematology toxicity and Grade 4 hematology toxicity, febrile neutropenia or infection, and/or thrombocytopenia associated with bleeding, the dosage of cytotoxic agents in the next cycle was reduced by $25 \%$.

Patients were subsequently divided as good responders and poor responders. Patients achieving complete response or partial response were defined as "responders", and patients with stable disease or progressive disease were defined as "non-responders".

All patients were followed up every 2 months until death or the end of the study period by telephone and inpatient and outpatient clinical medical records. Overall survival (OS) was regarded as the date of diagnosis to the date of death, and progression-free survival (PFS) was evaluated from the date of diagnosis to the time of progression or death without progression. All patients were followed up until November 2011.

\section{Genotyping}

All participants were asked to provide $5 \mathrm{~mL}$ blood, and the blood samples were stored at $-20^{\circ} \mathrm{C}$. Genomic DNA was extracted using the Qiagen Blood Kit (Qiagen, Chastworth, CA, USA) according to manufacturer instructions. Polymerase chain reaction (PCR) combined with a restriction fragment length polymorphism assay was used for genotyping. The XRCC1 Arg194Trp, XRCC1 Arg280His, XRCC1 Arg399Gln, ERCC5 His1104Asp, and ERCC5 His46His gene polymorphisms were identified as in previous studies (Kalikaki et al., 2009). The primers and probes of XRCC1 Arg194Trp, XRCC1 Arg399Gln, ERCC5 His1104Asp, and ERCC5 His46His were designed using the Sequenom Assay Design 3.1 software (Sequenom, San Diego, CA, USA) (Table 1). The PCR was conducted using $5 \mathrm{ng}$ genomic DNA in a $5-\mu \mathrm{L}$ reaction in the GeneAmp ${ }^{\circledR}$ PCR System 9700 with Dual 384-Well Sample Block Module (Applied Biosystems, Carlsbad, CA, USA). The shrimp alkaline phosphatase enzyme solution (Sequenom) was added to remove excess dNTPs, and iPLEX ${ }^{\circledR}$ Gold SBE chemistry (Sequenom) was conducted to perform the base extension reaction. The CLEAN resin (Sequenom) was used to produce the final base extension 
products. A total of 10-nL reaction solution was used for a 384 format SpectroCHIP microarray (Sequenom). The MassARRAY Analyzer Compact with ACQUAIRE Module (Sequenom) acquired spectra from the SpectroCHIP, and spectral data were automatically obtained and saved to the MassARRAY database. For quality control, we randomly selected $10 \%$ of the cases and controls to genotype again by different researchers. The reproducibility was $100 \%$.

Table 1. Primers for genotyping XRCC1 and ERCC5.

\begin{tabular}{lllll}
\hline Genotype & $\begin{array}{l}\text { Single nucleotide } \\
\text { polymorphism }\end{array}$ & Alleles & Primer sequence (5'-3') & Probe sequence (5'-3') \\
\hline XRCC1 Arg194Trp & rs1799782 & C/T & GCCAGGGCCCCTCCTTCAA & TACCCTCAGACCCAC GAGT \\
XRCC1 Arg399Gln & rs25487 & G/A & TTGTGCTTTCTCTGTGTCCA & TCCTCCAGCCTTTTC TGATA \\
ERCC5 His1104Asp & rs17655 & G/C & TTACGTCTTTGCGACAAATTCATT & CATTAAAGATGAACTTTCAGCAT \\
ERCC5 His46His & rs1047768 & C/T & AGCCTCGCCTTTGCCGAT & CTTCTGACCCATGCCCACC \\
\hline
\end{tabular}

\section{Statistical analysis}

All analyses were performed with the SPSS Version 16.0 software (SPSS Inc., Chicago, IL, USA). Continuous variables are reported as means \pm standard deviation (SD), while categorical variables are shown as frequencies and percentages. Demographic and clinical variables were compared across genotypes using the chi-squared test. The association of polymorphisms of genotypes with response to chemotherapy was calculated using the odds ratios (OR) with a corresponding 95\% confidence interval (CI). Our primary end point was OS, which was calculated as the time from diagnosis until death from any cause, or the last known date alive. PFS following treatment was calculated from the initiation of therapy to the first recorded date of progression, death, or last follow-up evaluation. The association between the four SNPs and OS and PFS of NSCLC was estimated using Cox's proportional hazard model, for which the most frequent genotype was used as the reference group. Statistical significance was defined as a two-sided $\mathrm{P}$ value of less than 0.05 .

\section{RESULTS}

The clinic pathological characteristics of the 378 patients included in the study are shown in Table 2. All 378 NSCLC patients received platinum-based chemotherapy as first-line treatment, and 226 of these patients died during the follow-up period. The median age of the cases was $62.4 \pm 6.5$ years, and ranged from 36 to 78 years. A total of $297(78.6 \%)$ patients were males. Older patients had a higher risk of death from NSCLC. The majority of patients presented at clinical stages III to IV of the disease $(72.5 \%)$, and patients at a higher clinical stage had an increased risk of death from NSCLC $(\mathrm{P}<0.05)$. A total of $216(57.1 \%), 125$ (33.1\%), and 37 (9.8\%) patients had the histological-type adenocarcinoma, -carcinoma, and other, respectively. Two hundred and twelve $(56.1 \%)$ patients had a smoking habit.

Among the 378 patients, 144 patients (38.1\%) showed a good response to platinumbased chemotherapy, and the remaining 234 showed a poor response to chemotherapy (Table 3). We found a significant difference between response in terms of the XRCC1 Arg399Gln and ERCC5 His46His polymorphisms $(\mathrm{P}<0.05)$. Individuals with the XRCC1 399A/A genotype were more likely to show a good response to chemotherapy, with an OR $(95 \% \mathrm{CI})$ of 2.27 
(1.64-6.97). Similarly, the ERCC5 46T/T genotype was significantly associated with a good response to platinum-based chemotherapy, with an OR $(95 \% \mathrm{CI})$ of $1.90(1.10-3.28)$.

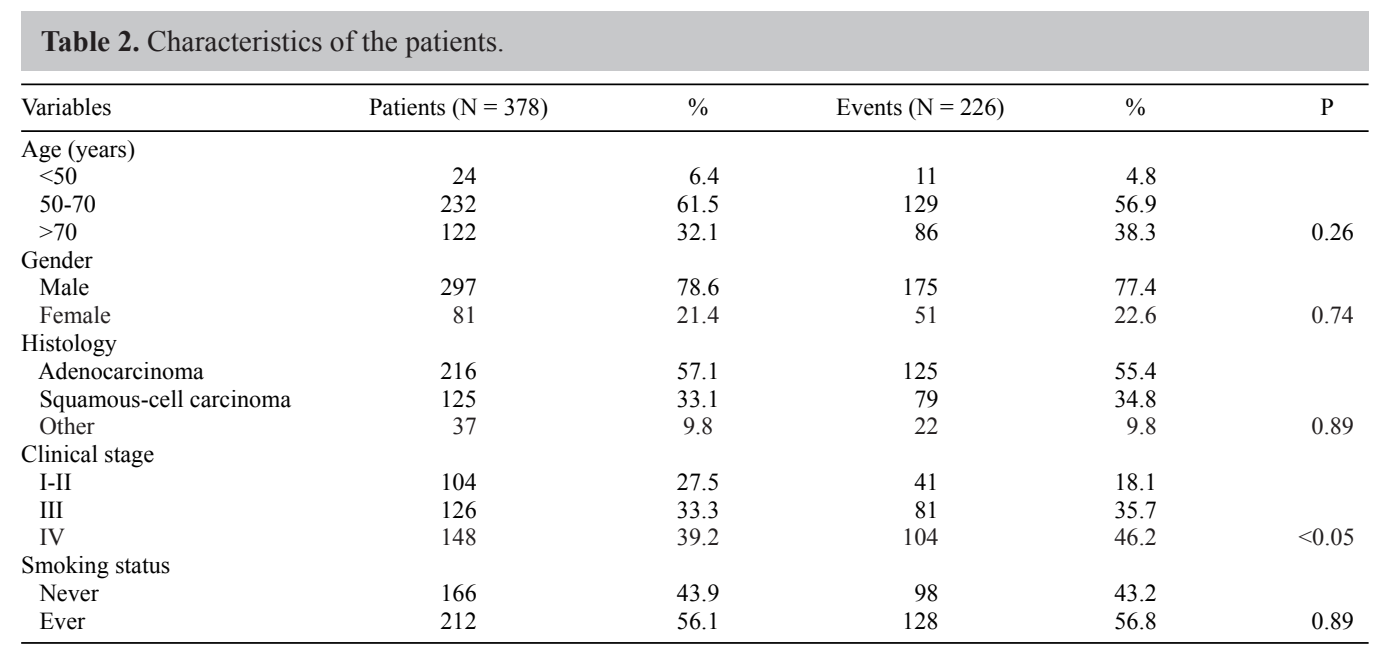

Table 3. Distribution of polymorphisms in four SNPs and response to platinum-based chemotherapy.

\begin{tabular}{|c|c|c|c|c|c|c|c|}
\hline \multirow[t]{2}{*}{ Genotypes } & \multicolumn{2}{|c|}{ Total } & \multicolumn{2}{|c|}{ Good response } & \multicolumn{2}{|c|}{ Poor response } & \multirow[t]{2}{*}{ OR $(95 \% \mathrm{CI})$} \\
\hline & $(\mathrm{N}=378)$ & $\%$ & $(\mathrm{~N}=144)$ & $\%$ & $(\mathrm{~N}=234)$ & $\%$ & \\
\hline \multicolumn{8}{|c|}{ XRCC1 Arg194Trp } \\
\hline $\mathrm{C} / \mathrm{C}$ & 205 & 54.2 & 71 & 49.2 & 134 & 57.3 & - \\
\hline $\mathrm{C} / \mathrm{T}$ & 119 & 31.5 & 48 & 33.2 & 71 & 30.5 & $1.28(0.78-2.09)$ \\
\hline $\mathrm{T} / \mathrm{T}$ & 54 & 14.3 & 25 & 17.6 & 29 & 12.3 & $1.63(0.84-3.12)$ \\
\hline \multicolumn{8}{|c|}{ XRCC1 Arg399Gln } \\
\hline $\mathrm{G} / \mathrm{G}$ & 171 & 45.2 & 52 & 36.3 & 119 & 50.7 & - \\
\hline $\mathrm{G} / \mathrm{A}$ & 160 & 42.3 & 64 & 44.1 & 96 & 41.2 & $1.53(0.94-2.57)$ \\
\hline $\mathrm{A} / \mathrm{A}$ & 47 & 12.5 & 28 & 19.6 & 19 & 8.1 & $2.27(1.64-6.97)$ \\
\hline \multicolumn{8}{|c|}{ ERCC5 His46His } \\
\hline $\mathrm{C} / \mathrm{C}$ & 142 & 37.6 & 45 & 31.5 & 97 & 41.4 & - \\
\hline $\mathrm{C} / \mathrm{T}$ & 128 & 33.9 & 48 & 33.6 & 80 & 34.1 & $1.29(0.76-2.21)$ \\
\hline $\mathrm{T} / \mathrm{T}$ & 108 & 28.5 & 51 & 34.9 & 57 & 24.6 & $1.90(1.10-3.28)$ \\
\hline \multicolumn{8}{|c|}{ ERCC5 His1104Asp } \\
\hline $\mathrm{C} / \mathrm{C}$ & 139 & 36.8 & 49 & 34.3 & 90 & 38.3 & - \\
\hline $\mathrm{C} / \mathrm{G}$ & 165 & 43.7 & 63 & 43.8 & 102 & 43.6 & $1.13(0.69-1.86)$ \\
\hline $\mathrm{G} / \mathrm{G}$ & 74 & 19.5 & 32 & 21.9 & 42 & 18 & $1.40(0.75-2.59)$ \\
\hline
\end{tabular}

A multivariate analysis was performed to determine the association between the four SNPs and the PFS and OS of NSCLC (Table 4). The XRCC1 399A/A genotype was significantly associated with a longer PFS and OS, and the hazard ratios (HRs) $(95 \% \mathrm{CI})$ were $0.48(0.25-0.88)$ and $0.51(0.26-0.98)$, respectively. Moreover, NSCLC patients carrying the ERCC5 46T/T genotype were more likely to have higher PFS and OS as a result of platinumbased chemotherapy, and the HRs $(95 \% \mathrm{CI})$ were 0.47 (0.22-0.82) and $0.52(0.31-0.96)$, respectively. However, we did not find any association between the polymorphisms XRCC1 Arg194Trp and ERCC5 His1104Asp with NSCLC prognoses. 
Table 4. Distribution of polymorphisms in four SNPs and response to platinum-based chemotherapy.

\begin{tabular}{|c|c|c|c|c|c|c|c|c|}
\hline & \multicolumn{4}{|c|}{ Disease-free survival } & \multicolumn{4}{|c|}{ Overall survival } \\
\hline & Events $(\mathrm{N}=271)$ & $\%$ & $\mathrm{HR}(95 \% \mathrm{CI})^{1}$ & $\mathrm{P}$ & Events $(\mathrm{N}=226)$ & $\%$ & HR $(95 \% \mathrm{CI})^{1}$ & $\mathrm{P}$ \\
\hline \multicolumn{9}{|c|}{ XRCC1 Arg194Trp } \\
\hline $\mathrm{C} / \mathrm{C}$ & 154 & 56.8 & - & & 130 & 57.5 & - & \\
\hline $\mathrm{C} / \mathrm{T}$ & 85 & 31.4 & $0.95(0.66-1.37)$ & 0.77 & 68 & 30.1 & $0.91(0.61-1.33)$ & 0.58 \\
\hline $\mathrm{T} / \mathrm{T}$ & 32 & 11.8 & $0.79(0.47-1.31)$ & 0.34 & 28 & 12.4 & $0.82(0.47-1.39)$ & 0.43 \\
\hline \multicolumn{9}{|c|}{ XRCC1 Arg399Gln } \\
\hline $\mathrm{G} / \mathrm{G}$ & 143 & 52.7 & - & & 115 & 51.2 & - & \\
\hline $\mathrm{G} / \mathrm{A}$ & 109 & 40.2 & $0.81(0.58-1.15)$ & 0.22 & 94 & 41.4 & $0.87(0.60-1.24)$ & 0.41 \\
\hline $\mathrm{A} / \mathrm{A}$ & 19 & 7.1 & $0.48(0.25-0.88)$ & $<0.05$ & 17 & 7.4 & $0.51(0.26-0.98)$ & $<0.05$ \\
\hline \multicolumn{9}{|c|}{ ERCC5 His46His } \\
\hline $\mathrm{C} / \mathrm{C}$ & 119 & 43.8 & - & & 95 & 42.2 & - & \\
\hline $\mathrm{C} / \mathrm{T}$ & 91 & 33.7 & $0.85(0.58-1.24)$ & 0.37 & 75 & 33.1 & $0.88(0.58-1.31)$ & 0.21 \\
\hline$T / T$ & 61 & 22.5 & $0.47(0.22-0.82)$ & $<0.05$ & 56 & 24.7 & $0.52(0.31-0.96)$ & $<0.05$ \\
\hline \multicolumn{9}{|c|}{ ERCC5 His1104Asp } \\
\hline $\mathrm{C} / \mathrm{C}$ & 107 & 39.5 & - & & 87 & 38.5 & & \\
\hline $\mathrm{C} / \mathrm{G}$ & 117 & 43.2 & $0.92(0.64-1.32)$ & 0.64 & 97 & 42.9 & $0.94(0.64-1.38)$ & 0.73 \\
\hline $\mathrm{G} / \mathrm{G}$ & 47 & 17.3 & $0.83(0.52-1.32)$ & 0.35 & 42 & 18.6 & $0.90(0.55-1.47)$ & 0.67 \\
\hline
\end{tabular}

${ }^{1}$ Adjusted for age, gender, histology, clinical stage, and smoking status.

\section{DISCUSSION}

In the current study, we examined whether polymorphisms in four SNPs were related to the metabolism of platinum-based regimens and could be used as predictors of clinical outcome of NSCLC patients treated with platinum-based chemotherapy.The NER pathway functions in repairing bulky, helix-distorting adducts, which are formed by cisplatin and its analogs (Kelland, 2000; Kartalou and Essigmann, 2001). The cisplatin-DNA adducts could be removed by platinum-resistant cells during the NER pathway and thus escape apoptosis. Results of the present study indicated that NSCLC patients carrying the XRCC1 399A/A genotype had a higher probability of showing a good response to platinum-based treatment compared to those with the G/G genotype. The PFS and OS of patients with the A/A genotype were significantly longer than those of the $\mathrm{G} / \mathrm{G}$ genotype $(\mathrm{PFS}$ : $\mathrm{HR}=0.48,95 \% \mathrm{CI}=$ 0.25-0.88; OS: $\mathrm{HR}=0.51,95 \% \mathrm{CI}=0.26-0.98$ ). Polymorphisms of XRCC1 Arg399Gln were shown to be associated with higher levels of DNA adducts, suggesting a deficiency in the DNA repair function, and a better efficacy of platinum-based chemotherapy (Lunn et al., 1999; Duell et al., 2000). Previous studies have shown that polymorphisms in XRCC1 Arg399Gln were significantly associated with the prognosis of NSCLC; however, these results are inconsistent (Gurubhagavatula et al., 2004; Giachino et al., 2007; Sun et al., 2009; Yao et al., 2009). A study conducted in Taiwan reported that NSCLC patients with the XRCC1 399A/A genotype showed longer OS (Liao et al., 2012). However, two other studies conducted in Italy showed that XRCC1 $399 \mathrm{G} / \mathrm{G}$ was associated with marginally significant better survival among advanced NSCLC patients receiving cisplatin-based chemotherapy (Gurubhagavatula et al., 2004; Kalikaki et al., 2009). Two other studies conducted in eastern Chinese populations did not find any significant association between XRCC1 Arg399Gln and NSCLC (Sun et al., 2009; Yao et al., 2009). The inconsistency of these results could be explained by differences in genetic backgrounds, population background, source of controls, and sample size, or could be due to chance. Alternatively, gene-environment interactions may operate in the pathogenesis of NSCLC, and thus differences in environmental risk factors might affect NSCLC risk. In 
addition, we found a significant association between the polymorphism in ERCC5 His46His and clinical response among advanced NSCLC patients with platinum-based chemotherapy, and those carrying the ERCC5 46G/G genotype showed a significantly reduced risk of death from NSCLC and were more likely to show a good response to platinum-based chemotherapy. Several studies have assessed the association between ERCC5 His46His polymorphisms and prognosis and susceptibility of various cancers, such as endometrial cancer, head and neck cancer, and gastric cancer (Cheng et al., 2002; Carles et al., 2006; Hussain et al., 2009; Doherty et al., 2011). A study conducted in the USA that included 783 endometrial cancer cases and 795 controls indicated that ERCC5 46G/G was associated with a decreased risk of death from cancer (Cheng et al., 2002). Another study in China also indicated that ERCC5 $46 \mathrm{G} / \mathrm{G}$ was associated with reduced risk of gastric cancer (Hussain et al., 2009), and yet another study indicated that ERCC5 46G/G was associated with shorter time to progression and survival in advanced NSCLC (Carles et al., 2006). However, only one study has explored the association between the ERCC5 46G/G genotype and NSCLC, which indicated a significantly increased response to platinum-based chemotherapy treatment associated with the ERCC5 46G/G genotype (Sun et al., 2009), which was in accordance with results of the present study.

There were several limitations in our study. Although we adjusted for potential risk factors of prognosis, such as tumor histology, stage of disease, and smoking status, we did not collect data related to other clinical factors that could potentially influence the prognosis of NSCLC. Secondly, owing to the relatively small sample of patients, the association of XRCC1 Arg399Gln and ERCC5 His46His with the prognosis of NSCLC might have resulted from other environmental and genetic factors, and thus the validation of their association requires further analysis.

In summary, this study indicated that XRCC1 Arg399Gln and ERCC5 His46His polymorphisms might significantly influence the response to chemotherapy. These results suggest that these two polymorphisms should be routinely detected in NSCLC patients to determine patients who are more likely to benefit from chemotherapy. Further large studies are warranted to confirm the association and role of these genes in NSCLC.

\section{ACKNOWLEDGMENTS}

We are grateful for the help from the staff of the Third Affiliated Hospital of Harbin Medical University and to all patients who provided blood samples for our study.

\section{REFERENCES}

Carles J, Monzo M, Amat M, Jansa S, et al. (2006). Single-nucleotide polymorphisms in base excision repair, nucleotide excision repair, and double strand break genes as markers for response to radiotherapy in patients with Stage I to II head-and-neck cancer. Int. J. Radiat. Oncol. Biol. Phys. 66: 1022-1030.

Cheng L, Spitz MR, Hong WK and Wei Q (2000). Reduced expression levels of nucleotide excision repair genes in lung cancer: a case-control analysis. Carcinogenesis 21: 1527-1530.

Cheng L, Sturgis EM, Eicher SA, Spitz MR, et al. (2002). Expression of nucleotide excision repair genes and the risk for squamous cell carcinoma of the head and neck. Cancer 94: 393-397.

de Boer J and Hoeijmakers JH (2000). Nucleotide excision repair and human syndromes. Carcinogenesis 21: 453-460.

Doherty JA, Weiss NS, Fish S, Fan W, et al. (2011). Polymorphisms in nucleotide excision repair genes and endometrial cancer risk. Cancer Epidemiol. Biomarkers Prev. 20: 1873-1882.

Duell EJ, Wiencke JK, Cheng TJ, Varkonyi A, et al. (2000). Polymorphisms in the DNA repair genes XRCC1 and ERCC2 
and biomarkers of DNA damage in human blood mononuclear cells. Carcinogenesis 21: 965-971.

Giachino DF, Ghio P, Regazzoni S, Mandrile G, et al. (2007). Prospective assessment of XPD Lys751Gln and XRCC1 Arg399Gln single nucleotide polymorphisms in lung cancer. Clin. Cancer Res. 13: 2876-2881.

Grønberg BH, Bremnes RM, Flotten O, Amundsen T, et al. (2009). Phase III study by the Norwegian lung cancer study group: pemetrexed plus carboplatin compared with gemcitabine plus carboplatin as first-line chemotherapy in advanced non-small-cell lung cancer. J. Clin. Oncol. 27: 3217-3224.

Gurubhagavatula S, Liu G, Park S, Zhou W, et al. (2004). XPD and XRCC1 genetic polymorphisms are prognostic factors in advanced non-small-cell lung cancer patients treated with platinum chemotherapy. J. Clin. Oncol. 22: 2594-2601.

Hussain SK, Mu LN, Cai L, Chang SC, et al. (2009). Genetic variation in immune regulation and DNA repair pathways and stomach cancer in China. Cancer Epidemiol. Biomarkers Prev. 18: 2304-2309.

International Agency for Research on Cancer (IARC) (2008). Lung Cancer Incidence, Mortality and Prevalence Worldwide in 2008. Available at [http://globocan.iarc.fr/factsheet.asp]. Accessed January 30, 2013.

Jeon HS, Kim KM, Park SH, Lee SY, et al. (2003). Relationship between XPG codon 1104 polymorphism and risk of primary lung cancer. Carcinogenesis 24: 1677-1681.

Kalikaki A, Kanaki M, Vassalou H, Souglakos J, et al. (2009). DNA repair gene polymorphisms predict favorable clinical outcome in advanced non-small-cell lung cancer. Clin. Lung Cancer 10: 118-123.

Kartalou M and Essigmann JM (2001). Mechanisms of resistance to cisplatin. Mutat. Res. 478: 23-43.

Kelland LR (2000). Preclinical perspectives on platinum resistance. Drugs 59 (Suppl 4): 1-8.

Liao WY, Shih JY, Chang GC, Cheng YK, et al. (2012). Genetic polymorphism of XRCC1 Arg399Gln is associated with survival in non-small-cell lung cancer patients treated with gemcitabine/platinum. J. Thorac. Oncol. 7: 973-981.

Lunn RM, Langlois RG, Hsieh LL, Thompson CL, et al. (1999). XRCC1 polymorphisms: effects on aflatoxin B1-DNA adducts and glycophorin A variant frequency. Cancer Res. 59: 2557-2561.

Reed E (1998). Platinum-DNA adduct, nucleotide excision repair and platinum based anti-cancer chemotherapy. Cancer Treat. Rev. 24: 331-344.

Sun X, Li F, Sun N, Shukui Q, et al. (2009). Polymorphisms in XRCC1 and XPG and response to platinum-based chemotherapy in advanced non-small cell lung cancer patients. Lung Cancer 65: 230-236.

Tahara E, Yasui W, Ito H and Harris CC (2010). Recent progress in carcinogenesis, progression and therapy of lung cancer: the 19th Hiroshima Cancer Seminar: the 3rd Three Universities' Consortium International Symposium, November 2009. Jpn. J. Clin. Oncol. 40: 702-708.

Weaver DA, Crawford EL, Warner KA, Elkhairi F, et al. (2005). ABCC5, ERCC2, XPA and XRCC1 transcript abundance levels correlate with cisplatin chemoresistance in non-small cell lung cancer cell lines. Mol. Cancer 4: 18.

William WN Jr, Lin HY, Lee JJ, Lippman SM, et al. (2009). Revisiting stage IIIB and IV non-small cell lung cancer: analysis of the surveillance, epidemiology, and end results data. Chest 136: 701-709.

Yao CY, Huang XE, Li C, Shen HB, et al. (2009). Lack of influence of XRCC1 and XPD gene polymorphisms on outcome of platinum-based chemotherapy for advanced non small cell lung cancers. Asian. Pac. J. Cancer Prev. 10: 859-864. 